\title{
Perspectivas Conceituais e Instrumentos para Avaliação de Funcionalidade em Pacientes com Esquizofrenia
}

\author{
Luciana Amorim, Luciano Franzim Neto, Aline Battisti Archer, Juliana Frainer ${ }^{1}$, Roberto Moraes Cruz \\ Universidade Federal de Santa Catarina, Florianópolis-SC, Brasil
}

\section{RESUMO}

Pacientes diagnosticados com esquizofrenia apresentam déficits variados e podem necessitar de cuidados e acompanhamento constantes. Entender os aspectos da funcionalidade desses pacientes torna-se essencial para compreender o transtorno de forma mais completa. Para isso, é necessário identificar as perspectivas conceituais de funcionalidade e verificar os instrumentos disponíveis para avaliar esse fenômeno. Foi realizada uma revisão integrativa em artigos científicos nas línguas portuguesa e inglesa. Em relação às perspectivas conceituais, os resultados apontaram aspectos cognitivos e sintomas negativos enquanto variáveis preditoras da funcionalidade, e os aspectos constituintes do construto funcionalidade foram sintetizados em um modelo composto por dois domínios: objetivo e subjetivo. Foram encontrados 43 instrumentos utilizados para avaliar a funcionalidade em pacientes com esquizofrenia. Desses, 36 instrumentos, apesar de terem sido utilizados em pacientes com esquizofrenia, não eram específicos para esse contexto. Sugere-se que instrumentos sejam desenvolvidos e adaptados para o contexto dos pacientes com esquizofrenia levando em consideração o conhecimento e perspectivas conceituais já existentes na área.

Palavras-chave: avaliação psicológica; esquizofrenia; funcionalidade; instrumentos de medida.

ABSTRACT - Conceptual Perspectives and Instruments for Functional Evaluation in Patients with Schizophrenia Patients diagnosed with schizophrenia present varied deficits and may require constant care and company. Understanding the functional aspects of these patients becomes essential to understanding the disorder more fully. For this, the conceptual perspectives of functionality must be identified, and the instruments available to evaluate this phenomenon must be verified. An integrative review was conducted on scientific articles in Portuguese and English. Regarding the conceptual perspectives, the results pointed out cognitive aspects and negative symptoms as variables predicting functionality, and the constituent aspects of the construct functionality were synthesized in a model composed of two domains: objective and subjective. We found 43 instruments used to evaluate functionality in patients with schizophrenia. Of these, 36 instruments, although they were used in patients with schizophrenia, were not specific to this context. It is suggested that instruments be developed and adapted to the context of patients with schizophrenia taking into account the knowledge and conceptual perspectives already existing in the area.

Keywords: psychological assessment; schizophrenia; functionality; measuring instruments.

RESUMEN - Perspectivas Conceptuales e Instrumentos de Evaluación de Funcionalidad en Pacientes con Esquizofrenia Pacientes con diagnóstico de esquizofrenia presentan déficit variable y pueden requerir cuidado y acompañamiento constante. Es fundamental entender los aspectos de funcionalidad de esos pacientes para poder comprender el transtorno de esquizofrenia de forma más completa. Se necesitan identificar las perspectivas conceptuales de funcionalidad y verificar los instrumentos disponibles para evaluar ese fenómeno. Con este objetivo, fue realizada una revisión integrativa de artículos científicos en el idioma portugués e inglés. En cuanto a las perspectivas conceptuales, los resultados señalaron aspectos cognitivos y síntomas negativos, mientras que variables predictoras de la funcionalidad, y los aspectos que constituyen el constructo de funcionalidad, fueron sintetizados en un modelo compuesto por dos dominios: objetivo y subjetivo. Se encontraron 43 instrumentos utilizados para evaluar la funcionalidad en pacientes con esquizofrenia. Entre los instrumentos encontrados, 36 a pesar de haber sido utilizados en pacientes con esa enfermedad, no eran específicos para ese contexto. Se sugiere que los instrumentos deben desarrollarse y adaptarse al contexto de los pacientes con esquizofrenia teniendo en cuenta el conocimiento y las perspectivas conceptuales existentes en la literatura.

Palabras clave: evaluación psicológica; esquizofrenia; funcionalidad; instrumentos de medida.

A esquizofrenia pode ser considerada uma das dez maiores causas de incapacidade atingindo, pelo menos, 26 milhões de pessoas ao redor do mundo. Pacientes com esse diagnóstico apresentam dificuldades, como maiores índices de desemprego, baixa produtividade e necessidade de supervisão ou cuidado constantes (Fleischhacker et al., 2014; Moore, Harmell et al., 2013). Os sintomas típicos do transtorno (positivos: 
alucinação, delírio, comportamento e fala desorganizada; e negativos: expressão emocional diminuída e avolia) (American Psychiatric Association, 2013) são relacionados às dificuldades apresentadas pelos pacientes; entretanto, pesquisas que buscam identificar os preditores da incapacidade associada ao quadro de esquizofrenia apontam que apenas tais sintomas não explicam satisfatoriamente o desfecho de funcionalidade nos pacientes (Brandizzi et al., 2015; Fervaha, Foussias, Agid, \& Remington, 2015).

O processo de avaliação de funcionalidade e incapacidade acompanhou a mudança ocorrida com a introdução de medicações antipsicóticas nos anos 50, quando 95\% dos pacientes internados em hospitais psiquiátricos apresentaram redução nos sintomas típicos do transtorno e voltaram a participar da vida em comunidade, apresentando diferentes níveis de funcionamento social e ocupacional (Frese, Knight, \& Saks, 2009). A constatação da variação nos níveis de funcionamento e suas implicações na avaliação e o tratamento de pacientes com esquizofrenia evidenciaram a necessidade de aprimorar a definição do construto e de mensurá-lo. Atualmente, a funcionalidade em esquizofrenia pode ser entendida como um termo que engloba a participação do indivíduo em atividades cotidianas e na comunidade onde está inserido; de maneira similar, "incapacidade" é um termo que inclui a limitação ou restrição nas atividades cotidianas e na participação da vida em comunidade (Green, Llerena, \& Kern, 2015; World Health Organization \& Cássia Maria Buchalla (Ed.), 2003).

O Manual Diagnóstico e Estatístico de Transtornos Mentais (DSM) (American Psychiatric Association, 2013) e a Classificação Internacional de Doenças (CID) (World Health Organization, 2010a) são fontes utilizadas como referência para o diagnóstico de esquizofrenia (Ustun \& Kennedy, 2009) e onde são encontradas as primeiras definições de funcionalidade, sendo que o DSM teve influência direta no surgimento desse conceito. Enquanto a CID utiliza como critérios diagnósticos os sintomas típicos da esquizofrenia, desde a primeira edição do DSM, o diagnóstico da esquizofrenia aparece caracterizado pelo "empobrecimento das relações humanas" (American Psychiatric Association, 1952). Na terceira edição do Manual (American Psychiatric Association, 1980), os sintomas típicos não são mais critérios exclusivos de avaliação do transtorno, e a disfunção social e ocupacional aparecem como novo critério definidor da presença de esquizofrenia. Essa compatibilidade na descrição dos sintomas entre o DSM e a CID é compreensível, uma vez que ambas as classificações foram desenvolvidas paralelamente (Ustun \& Kennedy, 2009), porém, a inclusão da disfunção social e ocupacional, como critério no DSM, deixou aberta à avaliação dos profissionais clínicos os aspectos da funcionalidade na esquizofrenia, mesmo que, no manual, não tenha sido definido tal construto de maneira específica.
Em concordância à inclusão da disfunção social e ocupacional como novo critério definidor do transtorno, a partir da revisão da terceira edição do manual - DSMIII-R - foram incluídos alguns instrumentos de auxílio na avaliação do funcionamento dos pacientes (American Psychiatric Association, 1987). No DSM-III-R, foi incluído um instrumento de avaliação de funcionalidade: a Escala de Avaliação Global do Funcionamento (GAF), e em sua quarta edição revisada - DSM-IV-TR - foi acrescentada a Escala de Avaliação do Funcionamento Social e Ocupacional (SOFAS) (American Psychiatric Association, 2002). Ambos os instrumentos permaneceram como ferramentas para auxiliar a avaliação clínica da disfunção funcional ou ocupacional (funcionalidade) dos pacientes, até o lançamento da quinta versão do manual, em 2013, quando uma nova ferramenta foi introduzida: a Escala de Avaliação de Incapacidade da Organização Mundial da Saúde 2.0 (WHODAS 2.0) (World Health Organization, 2010b). A WHODAS 2.0 foi desenvolvida para avaliar o nível de funcionalidade em seis domínios de vida (cognição, mobilidade, autocuidado, convivência social, atividades de vida e participação na sociedade). A WHODAS 2.0 segue a definição proposta pela Classificação Internacional de Funcionalidade e Incapacidade (CIF) (Silveira et al., 2013; World Health Organization \& Cássia Maria Buchalla (Eds.), 2003) e inclui, nos seus domínios, a avaliação de aspectos de mobilidade e restrição física dos sujeitos.

Apesar da tentativa de introduzir ferramentas que auxiliem a avaliação de funcionalidade dos pacientes com esquizofrenia, ao comparar as descrições do critério diagnóstico B - disfunção social/ocupacional - no DSMIV-TR (American Psychiatric Association, 2002) e, na versão mais recente, o DSM-5, observa-se que não há mudanças na definição do construto Funcionalidade em esquizofrenia. Assim como nas edições anteriores, o DSM-5 se refere à disfunção social/ocupacional como um rebaixamento do funcionamento em uma ou mais áreas importantes da vida, como o trabalho, relações interpessoais ou autocuidado (American Psychiatric Association, 2013), e cita, como auxílio para referência, a comparação do indivíduo com esquizofrenia com irmãos não afetados pelo transtorno.

$\mathrm{Na}$ perspectiva psicométrica, a mensuração de um construto demanda que os aspectos ou atributos que o constituem sejam claramente delimitados, para que assim se possa definir operacionalmente quais são os indicadores comportamentais que o representam e possibilitam quantificá-los (Pasquali, 2010). No caso da avaliação de funcionalidade em esquizofrenia, as definições encontradas no DSM citam atributos que podem ser compreendidos como componentes do construto funcionalidade - participação do sujeito em atividades cotidianas e na comunidade onde está inserido em uma ou mais áreas importantes, como trabalho, relações interpessoais ou autocuidado - porém não indicam se 
estes são apenas alguns dos atributos do construto ou se representam a sua totalidade. Além disso, não delimitam que fatores constituem cada um desses possíveis atributos, não indicando, por exemplo, quais aspectos relacionados ao trabalho devem ser avaliados, dificultando a representação empírica do fenômeno funcionalidade em esquizofrenia.

Em relação aos instrumentos indicados nas diversas versões do Manual Diagnóstico e Estatístico de Transtornos Mentais - GAF, SOFAS e WHODAS 2.0 - uma fragilidade percebida é o fato desses instrumentos serem escalas genéricas de avaliação de funcionalidade. Essas escalas se propõem a avaliar a funcionalidade em uma ampla gama de transtornos mentais assim como em diferentes condições de saúde, sendo assim, podem incluir atributos irrelevantes à avaliação da funcionalidade no contexto específico da esquizofrenia e prejudicar a fidedignidade da avaliação dos pacientes com esse transtorno.

O objetivo deste artigo é caracterizar as perspectivas conceituais acerca da funcionalidade e os instrumentos disponíveis para avaliar esse fenômeno em indivíduos com esquizofrenia, com base nas evidências empíricas identificadas no estado da arte e nas perspectivas teóricas de investigação.

\section{Método}

Para caracterizar as perspectivas conceituais e os instrumentos de avaliação sobre funcionalidade em pacientes com esquizofrenia, elaborou-se uma revisão integrativa da literatura. Esse tipo de revisão permite uma síntese e um aprofundamento do conhecimento de determinado fenômeno, ou seja, de maneira sistemática e organizada, a revisão integrativa consiste na construção de uma análise ampla da literatura a fim de reunir e sintetizar resultados e conclusões de estudos realizados anteriormente (Benefield, 2003; Broome, 2000).

Foi realizada busca de artigos científicos nas línguas portuguesa e inglesa em bibliotecas virtuais e bases de dados gratuitas: SciELO, Biblioteca Virtual de Saúde (BVS), Schizophrenia Bulletin, Redalyc, Lilacs e Psycnet. Os critérios de seleção foram: 1. artigo publicado entre 2005 e 2016; 2. conter, no título, o termo "esquizofrenia" / "schizophrenia" associado aos termos "funcionalidade" / "functional outcome" / "functioning" / "everydayfunctioning" e "avaliação" / "medida" / "evaluation" / "trial / assessment"; 3. conter, nos artigos, instrumentos validados psicometricamente; 4. serem artigos que objetivassem a avaliação de funcionalidade de pacientes com esquizofrenia. Foram definidos como critérios de exclusão: 1. pesquisas que contivessem no título ou resumo as palavras "autismo"/"autismo" ou "demência"/“dementia”. Os Transtornos do Espectro Autista e a Esquizofrenia são síndromes diferentes quanto aos seus critérios de definição, idade de início e curso da doença. Entretanto, ambas apresentam aspectos que interferem na capacidade funcional desses pacientes: déficits nas funções sociais e emocionais e no processamento da informação (Eack et al., 2013; Lugnegard, Unenge Hallerback, Hjarthag, \& Gillberg, 2013). A esquizofrenia também compartilha algumas características com quadros de demência (Chan et al., 2015), que também impactam no funcionamento dos pacientes na vida cotidiana. Uma vez que a funcionalidade é estudada em quadros como autismo e demência, fez-se necessária a seleção de estudos que abordassem o tema apenas no contexto do paciente com esquizofrenia. Os outros três critérios de exclusão foram pesquisas que avaliassem: 2. aspectos estritamente fisiológicos ou neurológicos; 3. funcionalidade não associada à esquizofrenia; 4. aspectos cognitivos sem avaliação concomitante de funcionalidade.

Em seguida à realização da primeira seleção dos artigos, foram lidos e analisados aqueles que mencionaram e descreveram pelo menos um instrumento que avaliasse funcionalidade em sujeitos com esquizofrenia e/ou que explicitassem perspectivas conceituais referentes à funcionalidade na esquizofrenia. Os artigos que apresentaram todos os elementos de inclusão foram analisados nos seguintes aspectos: características das perspectivas conceituais do fenômeno funcionalidade na esquizofrenia, características estruturais e de utilização dos instrumentos de avaliação de funcionalidade, e dimensões do construto avaliadas nesse instrumento. Os artigos excluídos e aqueles que levantaram dúvidas em algum dos aspectos de inclusão foram revisados mais de uma vez respeitando o consenso dos autores.

\section{Resultados e Discussão}

A pesquisa nas bases de dados resultou em um total de 120 artigos, dos quais 42 foram coletados da Schizophrenia Bulletin, 56 da BVS, 6 da SciELO e 16 da Psycnet. Após a leitura, foram excluídos 52 artigos que apresentaram alguma limitação com relação ao escopo desta revisão. Dentre os 68 artigos restantes, apenas dois se referiam a estudos conduzidos no Brasil. Foram encontradas nove perspectivas conceituais em estudos de: Green, Kern, Braff e Mintz (2000), Gladsjo e colaboradores (2004), Brekke, Kay, Lee e Green (2005), Wykes e Reeder (2005), Bowie, Reichenberg, Patterson, Heaton e Harvey (2006), Granholm, Ben-Zeev e Link (2009), Harvey e Bellack (2009), Harvey (2014) e Campellone, Sanchez e Kring (2016).

\section{Perspectivas Conceituais de Funcionalidade em Pacientes Adultos com Esquizofrenia}

A revisão de literatura de Green et al. (2000) indicou como desfecho de funcionalidade alguns aspectos separados em três categorias gerais: 1. sucesso nos programas de 
reabilitação psicossocial, 2. estudos que acessaram habilidades de resolução de problemas sociais em ambiente de laboratório (capacidade funcional); e 3. estudos que consideraram desempenho em atividades cotidianas e aspectos gerais do comportamento em comunidade. Mesmo que sem a explicitação de matrizes teóricas que suportariam essas categorias gerais, a produção desse tipo de conhecimento é relevante por ser uma das primeiras iniciativas científicas para a organização da definição de funcionalidade.

A proposta de Gladsjo et al. (2004) foi desenvolvida para ajudar nas pesquisas de tratamentos farmacológicos focadas nos déficits cognitivos associados à esquizofrenia e destaca como indicadores de desfecho de funcionalidade: (a) mudanças no déficit cognitivo dos pacientes; (b) capacidade funcional; e, (c) aspectos cognitivos relacionados à funcionalidade cotidiana. Sugere também que, além desses indicadores, o histórico do paciente pode influenciar a funcionalidade.

Brekke et al. (2005) propuseram uma perspectiva conceitual de funcionalidade, testada por meio da análise fatorial, composta por cinco dimensões: Neurocognição, Cognição Social, Competência Social, Suporte Social e Funcionalidade Cotidiana. Wykes e Reeder (2005) sugeriram uma perspectiva cuja ênfase estava na cognição dos pacientes e também na relação entre habilidades cognitivas básicas e a funcionalidade cotidiana. Os fatores independentes que mediaram a relação cognição - funcionalidade foram: (a) oportunidade; (b) nível de habilidades pré-existentes; (c) suporte social; e (d) motivação.

Nas proposições de Bowie et al. (2006), foram indicadas quatro dimensões de funcionalidade: (a) desempenho neuropsicológico; (b) mensuração da capacidade funcional; (c) variação dos sintomas; (d) influências adicionais. Nesse estudo, assim como nos estudos de Brekke (et al., 2005) e Wykes e Reeder (2005), também foram testadas as dimensões da funcionalidade por meio de análise fatorial. Os resultados mostraram que o desempenho neuropsicológico influencia desfechos de funcionalidade cotidiana de forma mediada pela capacidade funcional e que sintomas não impactam diretamente nessa capacidade e no desempenho neuropsicológico. Mais especificamente, os autores sugeriram que sintomas negativos e depressão (e não os sintomas positivos) estavam relacionados com funcionalidade cotidiana, mas não com desempenho neuropsicológico ou medidas de capacidade funcional.

Granholm et al. (2009) relacionaram déficits neurocognitivos com capacidade funcional, medidos por instrumentos de performance (role-play), e afirmaram que estudos e avaliação da capacidade funcional são necessários, porém não são suficientes para explicar a funcionalidade cotidiana. Explicitaram, ainda, como mediadores dessa relação: (a) fatores pessoais (atitudes, anedonia, motivação, etc.) e, (b) fatores ambientais (suporte social, por exemplo).
Harvey e Bellack (2009) afirmaram que um dos maiores desafios relacionados ao estudo da funcionalidade em esquizofrenia continua sendo a falta de consenso sobre a definição do construto e de parâmetros que possam ser usados para classificar o processo de recuperação dos pacientes. Eles propuseram a constituição da funcionalidade em três dimensões baseadas nas atividades cotidianas que são requisitos para um funcionamento adaptado dentro das culturas ocidentais: (a) atividades produtivas; (b) atividades de automanutenção ou cuidado e de habitação; e (c) relações interpessoais. Entenderam, também, que essas três amplas dimensões são relevantes para qualquer indivíduo inserido nessa cultura e ainda sugeriram uma avaliação de funcionalidade em termos de nível e amplitude. Harvey (2014) revisou as dimensões de funcionalidade na esquizofrenia e classificou em déficits objetivos e subjetivos. Déficits objetivos de funcionalidade são os comportamentos observáveis dos pacientes nas seguintes áreas: (a) ocupacional ou de trabalho; (b) relações interpessoais; e (c) autocuidado. Os déficits subjetivos são as impressões percebidas pelo paciente em relação à: (a) sobrecarga ocasionada pela doença; e (b) qualidade de vida. Suas conclusões são um apanhado das perspectivas teóricas estudadas na última década e organizam grande parte das dimensões que atualmente são compreendidas enquanto componentes da funcionalidade na esquizofrenia.

A meta-análise de Campellone, Sanchez e Kring (2016) discutiu um modelo que investiga o mecanismo pelo qual sintomas negativos colaboram para um baixo nível de funcionalidade na esquizofrenia. O estudo buscou avaliar a associação entre sintomas negativos e crenças de desempenho derrotistas (CDD) ou pensamentos negativos sobre sua própria capacidade - um dos fatores do atributo motivação -, evidenciando a influência de tal fator da motivação sobre a inibição de comportamentos dirigidos à metas e engajamento dos sujeitos em atividades cotidianas.

Cada perspectiva teórica de funcionalidade em pacientes adultos com esquizofrenia explicita ou enfatiza um aspecto do fenômeno funcionalidade. Ao considerar funcionalidade de pacientes com esquizofrenia, como o funcionamento do indivíduo nas atividades cotidianas e a sua participação na sociedade e o baixo grau de funcionalidade enquanto deficiências, limitação da atividade ou restrição na participação do sujeito na sociedade (World Health Organization \& Cássia Maria Buchalla (Ed.), 2003), é possível perceber que as variáveis destacadas nos estudos encontrados, nesta revisão de literatura, possuem diferentes papéis em relação à funcionalidade em esquizofrenia. Na Figura 1, é proposta um modelo que sintetiza as principais variáveis preditoras do construto funcionalidade em pacientes com esquizofrenia, bem como os domínios e atributos que o compõem. 


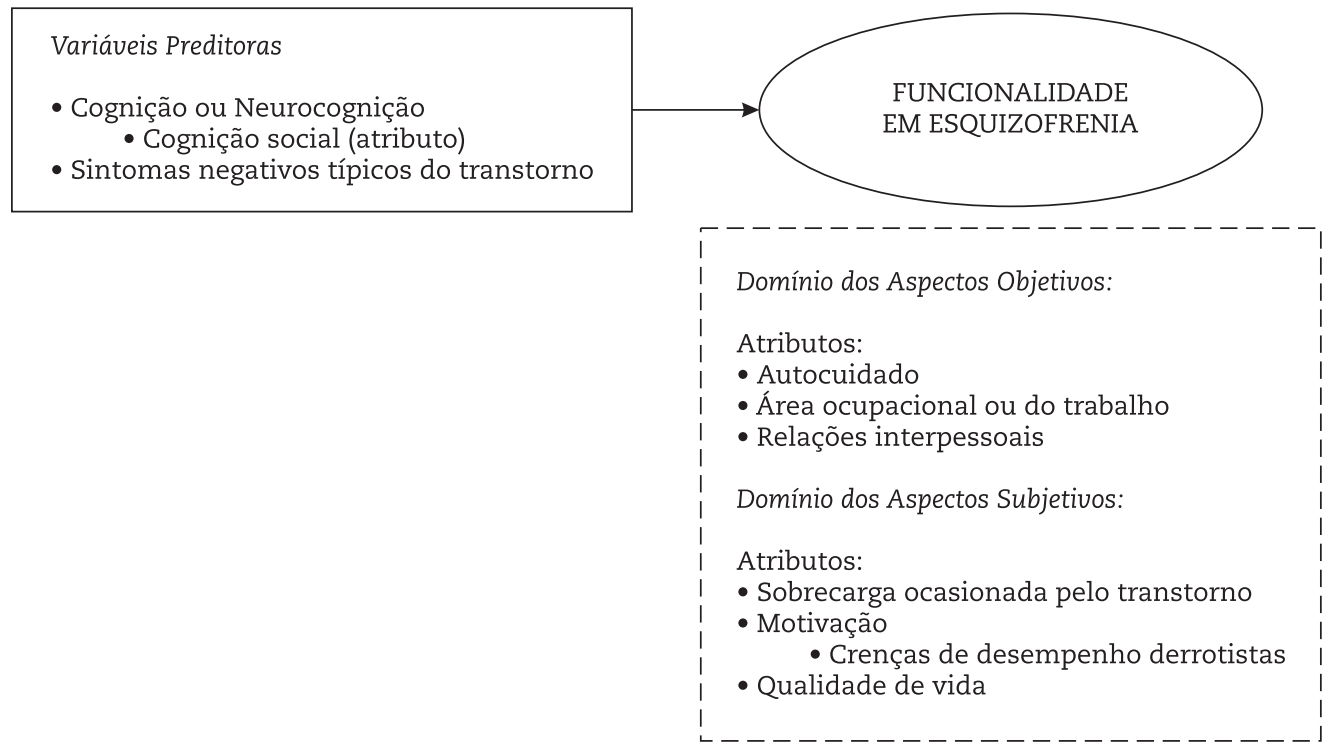

Figura 1. Modelo síntese das variáveis preditoras, domínios e atributos do construto funcionalidade em esquizofrenia

Todos os estudos levantados apontaram aspectos intrínsecos aos sujeitos como sintomas negativos e a neurocognição ou a cognição social (um de seus atributos) enquanto variáveis preditoras da funcionalidade em esquizofrenia (Bowie et al., 2006; Brekke et al., 2005; Campellone et al., 2016; Gladsjo et al., 2004; Granholm et al., 2009; Green et al., 2000; Harvey, 2014; Harvey \& Bellack, 2009; Wykes \& Reeder, 2005). De uma forma geral, os artigos estudados, nesta revisão, apontaram, enquanto aspectos intrínsecos dos indivíduos, atributos importantes da funcionalidade em esquizofrenia: as atividades de autocuidado, a ocupação ou trabalho e as relações interpessoais (Bowie et al., 2006; Brekke et al., 2005; Campellone et al., 2016; Gladsjo et al., 2004; Granholm et al., 2009; Green et al., 2000; Harvey, 2014; Harvey \& Bellack, 2009; Wykes \& Reeder, 2005), agrupados por Harvey (2014) no que se pode denominar de domínio dos aspectos objetivos. Entretanto, nenhum dos estudos indicou quais seriam fatores e facetas que compõem cada um dos atributos desse domínio. Um outro agrupamento proposto por Harvey pode ser entendido como o domínio dos aspectos subjetivos e é composto por outros dois atributos do construto: sobrecarga ocasionada pelo transtorno e qualidade de vida. A motivação dos pacientes parece ser um dos fatores do atributo sobrecarga ocasionada pelo transtorno (Campellone et al., 2016; Granholm et al., 2009; Harvey, 2014; Wykes \& Reeder, 2005) e as crenças de desempenho derrotistas (CDD) uma das facetas que compõem o fator Motivação (Campellone et al., 2016).

Ao relacionar o modelo que sintetiza as evidências empíricas sobre o construto estudado e uma das principais referências de diagnóstico clínico, o DSM, é possível observar que, no manual, estão caracterizadas, de modo incompleto e impreciso, as variáveis que constituem a funcionalidade, mesmo que esse construto seja considerado um indicador da esquizofrenia. No DSM, é explicitada a existência de uma relação entre sintomas típicos e os possíveis prejuízos no funcionamento social e/ou ocupacional e no autocuidado do paciente com esquizofrenia, embora tais prejuízos não sejam caracterizados. A falta de delimitação atributos da funcionalidade no DSM-5 faz com que a avaliação do fenômeno dependa de que o clínico ou o entrevistador conheçam a história prévia do paciente ou que façam uso de um instrumento de avaliação de funcionalidade genérico proposto pelo manual (American Psychiatric Association, 2013). Nesse sentido, com base na Figura 1, para tornar a avaliação do paciente com esquizofrenia mais completa, é necessário que o clínico e o pesquisador tenham clareza de que, quando avaliadas variáveis como sintomas positivos e negativos e fenômenos clínicos relacionados, está-se caracterizando a esquizofrenia, mas não a funcionalidade. Avaliar a funcionalidade de pacientes com esquizofrenia envolve avaliar mais do que os sintomas típicos desse transtorno e apenas alguns aspectos sociais, significa avaliar os sintomas em relação às variáveis que constituem a funcionalidade, como autocuidado, relações interpessoais, desempenho ocupacional, sobrecarga ocasionada pelo transtorno e qualidade de vida.

Em síntese, quando são realizadas avaliações da funcionalidade em pacientes com esquizofrenia, é necessário também considerar as evidências empíricas disponíveis na literatura científica, uma vez que elas podem auxiliar no avanço do conhecimento registrado nas principais referências da área clínica (CID e DMS-5), de modo a não 
restringir a análise do fenômeno à mera descrição dos sintomas, de fenômenos clínicos associados ao transtorno ou de apenas algumas dimensões da funcionalidade. Avaliar a funcionalidade em pacientes com esquizofrenia exige do profissional clareza a respeito das variáveis constituintes da esquizofrenia, dos fenômenos clínicos associados ao transtorno que influenciam a funcionalidade e das variáveis que de fato constituem o construto funcionalidade em esquizofrenia. Dessa forma, será possível uma avaliação mais fidedigna desse fenômeno.

\section{Instrumentos para Avaliação de Funcionalidade em Pacientes Adultos com Esquizofrenia}

Foram encontrados 43 instrumentos utilizados para avaliar a funcionalidade em pacientes com esquizofrenia. Destes, 36 instrumentos, apesar de terem sido usados em pacientes com esquizofrenia, não eram específicos para esse contexto. Apesar da quantidade de instrumentos utilizados para avaliar a funcionalidade em pacientes com esquizofrenia, encontrados nesta revisão, existem diferenças entre a abrangência dos construtos medidos nos instrumentos (Gould, Sabbag, Durand, Patterson, \& Harvey, 2013; Keefe, Poe, Walker, \& Harvey, 2006). De modo geral, há três classes de construtos medidos por instrumentos utilizados em pesquisas, nas quais foi realizada a avaliação de funcionalidade de pacientes com esquizofrenia. São elas: 1. sintomas psiquiátricos de forma genérica (avaliação global); 2. funcionalidade de pacientes com transtornos psiquiátricos em geral ou com outra condição clínica incapacitante; e 3 . funcionalidade de pacientes com esquizofrenia.

A Global Assessment Scale (GAS) é um exemplo de instrumento de avaliação global e resultado de um aperfeiçoamento da primeira escala de avaliação global Health Sickness Rating Scale (HSRS) - desenvolvida em 1962 (Thornicroft \& Tansella, 2010). Dentre os domínios que compõem a estrutura dessas escalas, são avaliados sintomas psiquiátricos, como humor depressivo, insônia, euforia e comportamento antissocial. Apesar de ambas, HSRS e GAS, estarem em desuso por apresentarem versões mais recentes, nesta revisão, três estudos utilizaram a GAS (Eack, Pogue-Geile, Greenwald, Hogarty, \& Keshavan, 2011; Grant, Huh, Perivoliotis, Stolar, \& Beck, 2012; MacDonald et al., 2005). O ensaio clínico de Grant et al. (2012), por exemplo, buscou avaliar a eficácia da terapia cognitiva em pacientes com esquizofrenia com baixo nível de funcionamento e utilizou a GAS como único instrumento de avaliação de funcionalidade, além de dois outros instrumentos para avaliar sintomas típicos do transtorno. Levando em conta que a GAS também avalia sintomas típicos do transtorno, qualquer resultado do estudo precisaria ser interpretado com ressalvas (Bellack et al., 2007), ou seja, afirmar que os sujeitos pesquisados apresentaram melhora em seu funcionamento é inadequado, uma vez que a funcionalidade não foi avaliada pelos instrumentos. Os autores, inclusive, reconheceram que a escolha da GAS para avaliar a funcionalidade é passível de crítica, e necessita ser repensada no caso de estudos posteriores.

A Global Assessment of Functioning (GAF), usada em nove estudos encontrados nesta revisão, foi projetada como parte da avaliação do eixo 5 no DSM-III-R (American Psychiatric Association, 1987) e continua a ser amplamente utilizada. Tanto quanto a GAS, apresenta como principal limitação a combinação da avaliação de sintomas e aspectos de funcionalidade resultando em um único escore. Numa tentativa de minimizar a indiferenciação entre sintoma e funcionalidade, o DSM-IV (American Psychiatric Association, 2002) excluiu os itens relacionados a sintomas psiquiátricos dos domínios da GAF, construindo outra escala denominada Social and Ocupational Functioning Assessment Scale (SOFAS). A SOFAS foi sugerida como um recurso para monitorar a funcionalidade social e ocupacional independentemente da severidade dos sintomas do transtorno e apresentou adequada validade psicométrica (Thornicroft \& Tansella, 2010). Entretanto, os dados encontrados nesta revisão evidenciam que a SOFAS é pouco utilizada no contexto da pesquisa, aparecendo em apenas três estudos clínicos nos últimos 10 anos. De modo a corroborar esse achado, no estudo de Gorwood, Burns, Juckel, Rossi, San e Hargarter (2013) acerca do uso de instrumentos padronizados no contexto clínico, dentre 4163 psiquiatras consultados, $47 \%$ dos sujeitos entrevistados relataram o uso da GAF como instrumento de avaliação de sua preferência, sendo que apenas $9 \%$ faz uso da SOFAS.

A Global Functioning: Social Scale (GF: social), Global Functioning: Role (GF: Role), Global Assessment of Functioning: Disability (GAF-D: disability) resultam de esforços para discriminar domínios dos sintomas avaliados na GAF (Cornblatt et al., 2007). Por meio dela, é avaliada a funcionalidade de pacientes com transtornos psiquiátricos em geral. Os itens desses instrumentos foram retirados da GAF, indo ao encontro de perspectivas conceituais de incapacidade, tal como apresentada na CIF, em que há uma clara distinção entre sintomas e funcionalidade (Harvey, 2014).

Outra escala que avalia globalmente a funcionalidade de pacientes e que não distingue a sintomatologia dos transtornos ou de outras condições médicas de funcionamento psicológico é a World Health Organization Psychiatric Disability Assessment Schedule (WHODAS 2.0). Esse instrumento tem sido utilizado em estudos conduzidos em populações com transtornos mentais e indicado, no DSM-5, em substituição à GAF (American Psychiatric Association, 2013). Apesar de excluir a avaliação de sintomas e se concentrar na avaliação da funcionalidade - um avanço em relação à sua antecessora, a GAF - a WHODAS 2.0 é uma medida genérica usada para avaliar um amplo espectro de transtornos em uma variedade de situações, em diferentes grupos ou populações. 
Instrumentos como GAS, GAF e WHODAS 2.0, que avaliam vários tipos de transtornos, os quais podem afetar a funcionalidade, trazem resultados incertos quando mensuram aspectos específicos de um transtorno como a esquizofrenia. Instrumentos que avaliam, de forma global várias dimensões de diferentes transtornos mentais ou condições físicas, podem produzir vieses de interpretação na aferição de dificuldades de interação social comuns em pacientes com esquizofrenia. Além disso, não ajudam o pesquisador na compreensão de quais especificidades apresentam maior impacto preditor de funcionalidade desses pacientes. É necessária, portanto, uma medida capaz de avaliar pequenas variações e seus possíveis impactos na funcionalidade (Thornicroft \& Tansella, 2010).

$\mathrm{Na}$ Tabela 1, podem ser observados sete instrumentos, cujo construto avaliado é a funcionalidade em pacientes com esquizofrenia. Esses instrumentos, desenvolvidos ou adaptados para uso com pacientes com esquizofrenia, apresentaram estudos de validade de conteúdo, ou seja, houve um cuidado por parte dos pesquisadores para que os itens dos instrumentos fossem compreensíveis ao seu público-alvo. Instrumentos que avaliam a funcionalidade por meio de situações e dificuldades cotidianas típicas dos pacientes com esquizofrenia são imprescindíveis. Questões de validade semântica e a clareza na construção dos itens do instrumento terão impacto quando a amostra estudada apresenta dificuldades cognitivas que interferem diretamente na capacidade de interpretação do conteúdo dos itens (Pasquali, 2010; Pontes \& Elkis, 2013).

Especialmente para o exame dos sete instrumentos de avaliação de funcionalidade em pacientes com esquizofrenia, foram consideradas dois grandes domínios constituintes da funcionalidade: 1. aspectos objetivos e 2. aspectos subjetivos. Os aspectos objetivos são: déficit ocupacional, funcionamento social ou relações interpessoais e autocuidado. Os aspectos subjetivos são a sobrecarga ocasionada pelo transtorno e a percepção de qualidade de vida (Harvey, 2014).

Entre 2005 e 2016, a maioria das pesquisas utilizou instrumentos que priorizavam a dimensão objetiva da funcionalidade. Tal dimensão foi avaliada nos sete instrumentos da Tabela 1. O fator Relações Interpessoais foi avaliado em todos eles, seguido do fator Vida independente ou Autocuidado (seis ocorrências).

Tabela 1

Instrumentos para Avaliação de Funcionalidade em Pacientes Adultos com Esquizofrenia

\begin{tabular}{lll}
\hline \multicolumn{1}{c}{ Instrumentos } & \multicolumn{1}{c}{ Aspectos avaliados } & \multicolumn{1}{c}{ Estudos que utilizaram o instrumento } \\
\hline UPSA - University of California, & Autocuidado, relações & Bowie et al., 2007; Granholm et al., 2005; Harvey, \\
San Diego, Performance-Based & interpessoais, área & 2014; Heinrichs, Ammari, Miles, \& McDermid Vaz, \\
Skills Assessment & ocupacional & 2010; Keefe, Poe, Walker, Kang, \& Harvey, 2006; \\
& & Mausbach, Harvey, Goldman, Jeste, \& Patterson, \\
& & 2006; Moore, Harmell, et al., 2013; Moore, Viglione, \\
& Rosenfarb, Patterson, \& Mausbach, 2013
\end{tabular}

UPSA-B - Brief University of California, San Diego, Performance-Based Skills Assessment

SFS - Social Functioning Scale

QLS - Quality of Life Scale

\begin{abstract}
COTE - Comprehensive Occupational Therapy Evaluation Scale
\end{abstract}

RSAS - Revised Anhedonia Scale

LSP-VP-39 - Life Skills

Profile-Versão Portuguesa
Autocuidado, relações interpessoais

\begin{abstract}
Autocuidado, relações interpessoais, área ocupacional
\end{abstract}

Autocuidado, relações interpessoais, área ocupacional, qualidade de vida

Autocuidado, relações interpessoais, área ocupacional

Relações interpessoais

Autocuidado, relações interpessoais, área ocupacional
Cardenas et al., 2013; Gould et al., 2013; Mausbach et al., 2011; Mausbach, Moore, Bowie, Cardenas, \& Patterson, 2009; Pinkham, Penn, Green, \& Harvey, 2015; Sabbag et al., 2012; Shi, He, Cheung, Yu, \& Chan, 2013; Walther et al., 2016

Ciudad, Olivares, Bousono, Gomez, \& Alvarez, 2006; Mathews \& Barch, 2010

Bell, Corbera, Johannesen, Fiszdon, \& Wexler, 2013; Bowie et al., 2007; Cruz et al., 2016; Gould et al., 2013; Lysaker, Roe, \& Yanos, 2006; Velligan, Alphs, Lancaster, Morlock, \& Mintz, 2009; Zawadzki et al., 2013

Li, Ying, \& Deng, 2012

Granholm et al., 2009

Rocha C., Aguiar, \& Marques, 2008 
Dentre os instrumentos que avaliam aspectos objetivos da funcionalidade, destacam-se o Performance-Based Skills Assessment (UPSA) e a sua versão breve Brief University of California, San Diego, Performance-Based Skills Assessment - UPSA-B). Ambas as versões têm sido amplamente utilizadas nos últimos anos (Bowie et al., 2006; Thornicroft \& Tansella, 2010). A versão completa (UPSA), que foi elaborada para acessar habilidades necessárias para a vida cotidiana de pacientes adultos com esquizofrenia, abrange cinco áreas (cuidados domésticos, comunicação, finanças, transporte e planejamento de atividades de lazer). É um instrumento de medida baseado no desempenho observado em situações definidas, pontuado de acordo com critérios estabelecidos. A versão breve dessa escala contém apenas duas subescalas (Finanças e Comunicação) e tem apresentado altas correlações com outros instrumentos que acessam funcionamento global e desempenho cognitivo (Mausbach et al., 2008, 2006).

A dimensão subjetiva do construto Funcionalidade é considerada em menor quantidade de instrumentos de avaliação. A Qualidade de Vida, um aspecto da dimensão subjetiva da funcionalidade, foi avaliada por apenas um instrumento - Quality of Life Scale (QLS). Essa escala desenvolvida especificamente para pacientes com esquizofrenia, por Heinrichs, Hanlon e Carpenter (1984), e adaptada para o contexto brasileiro (Cardoso, Bandeira, Caiaffa, \& Fonseca, 2002).

Considerando os instrumentos que avaliam as dimensões de funcionalidade e o modelo que sintetiza as evidências empíricas levantadas neste estudo, é necessário que sejam produzidos e adaptados instrumentos que medem o conjunto de atributos constituintes do domínio dos aspectos subjetivos da funcionalidade, para além do fator qualidade de vida, seja acrescentando fatores dos atributos que compõem o domínio dos aspectos subjetivos em instrumentos já existentes ou criando instrumentos específicos para a avaliação desse domínio. Além disso, é importante que tais instrumentos possuam propriedades psicométricas adequadas, assim, o profissional que atua no contexto clínico e os pesquisadores da área da saúde mental terão uma avaliação mais fidedigna da funcionalidade em pessoas com esquizofrenia.

\section{Considerações Finais}

O objetivo deste estudo foi caracterizar as perspectivas conceituais de avaliação da funcionalidade em pacientes com esquizofrenia e verificar os instrumentos disponíveis para avaliar esse fenômeno. Em relação às perspectivas conceituais, é possível observar que estudiosos da área evidenciam tanto aspectos comuns, como diferentes em relação ao que caracteriza a funcionalidade de pacientes com tal transtorno. Como proposição, foi representado um modelo que sintetiza a relação entre variáveis preditoras da funcionalidade em pacientes com esquizofrenia e as variáveis constituintes desse fenômeno. Ter clareza dos limites e das relações entre essas variáveis é condição orientadora para atuação de profissionais da saúde mental e pesquisadores. O êxito do processo de avaliação dos indivíduos com esquizofrenia dependerá das estratégias de avaliação utilizadas pelo profissional, podendo priorizar o exame dos sintomas típicos da esquizofrenia, dos fenômenos clínicos relacionados ao transtorno, da funcionalidade de pacientes com esse transtorno ou, ainda, a relação entre esses três aspectos.

Nas pesquisas observadas que utilizam instrumentos de medida para avaliar a funcionalidade em pacientes com esquizofrenia, foi observado o uso de instrumentos que medem construtos com graus de abrangência variados em relação ao construto Funcionalidade. Há instrumentos que medem sintomas psiquiátricos de forma geral, outros medem a funcionalidade de paciente com transtornos psiquiátricos em geral ou ainda com outras condições clínicas incapacitantes e outros medem a funcionalidade de pacientes com esquizofrenia (sete de 43 instrumentos). Nesta revisão de literatura, também foi identificado que aspectos subjetivos da funcionalidade (impressões percebidas pelo paciente em relação à sobrecarga ocasionada pela doença e qualidade de vida) foram considerados com menor frequência nos instrumentos de medida, se comparado aos aspectos objetivos.

Com base no exame da literatura realizado, é preciso que estudos sejam conduzidos com a função de confirmar, ampliar ou propor representações que sintetizem evidências empíricas acerca do construto funcionalidade em esquizofrenia, possibilitando avanços na verificação científica dessas perspectivas e na delimitação da definição de funcionalidade e de seus domínios, atributos, fatores e facetas. Além disso, sugere-se que instrumentos sejam desenvolvidos e adaptados para o contexto dos pacientes com esquizofrenia levando em consideração o conhecimento e perspectivas conceituais já existentes na área. Futuras propostas conceituais e instrumentais podem incluir, com maior ênfase, aspectos subjetivos em seu escopo. É preciso, ainda, que a funcionalidade para a esquizofrenia seja pensada separadamente do conceito de funcionalidade global e que estudos confirmem essa diferença de construtos.

\section{Referências}

American Psychiatric Association (1952). Diagnostic and Statistical Manual of Mental Disorders. Washington: American Psychiatric Association Mental Hospital Service. 
American Psychiatric Association (1980). Diagnostic and Statistical Manual of Mental Disorders - Third Edition - DSM-III (3rd ed.). Washington: American Psychiatric Association.

American Psychiatric Association (1987). Diagnostic and Statistical Manual of Mental Disorders - Third Edition - Revised - DSM-III-R (3rd ed.). Washington: American Psychiatric Association.

American Psychiatric Association (2002). Diagnostic and Statistical Manual of Mental Disorders - Fourth Edition - Text Review - DSM-IV-TR. Washington: American Psychiatric Association.

American Psychiatric Association (2013). Diagnostic and Statistical Manual of Mental Disorders - Fifth Edition - DSM-5 (5th ed.). Washington: American Psychiatric Association.

Ascher-Svanum, H., Nyhuis, A. W., Faries, D. E., Kinon, B. J., Baker, R. W., \& Shekhar, A. (2008). Clinical, functional, and economic ramifications of early nonresponse to antipsychotics in the naturalistic treatment of schizophrenia. Schizophr Bull, 34(6), 1163-1171. doi: $10.1093 /$ schbul/sbm134

Bell, M. D., Corbera, S., Johannesen, J. K., Fiszdon, J. M., \& Wexler, B. E. (2013). Social cognitive impairments and negative symptoms in schizophrenia: are there subtypes with distinct functional correlates? Schizophr Bull, 39(1), 186-196. doi: 10.1093/schbul/sbr125

Bellack, A. S., Green, M. F., Cook, J. A., Fenton, W., Harvey, P. D., Heaton, R. K., ... Wykes, T. (2007). Assessment of community functioning in people with schizophrenia and other severe mental illnesses: A white paper based on an NIMH-sponsored workshop. Schizophrenia Bulletin, 33(3), 805-822. doi: 10.1093/schbul/sbl035

Benefield, L. E. (2003). Implementing evidence-based practice in home care. Home Healthc Nurse, 21(12), 801-804. Recuperado de http:// www.ncbi.nlm.nih.gov/pubmed/14665967

Bowie, C. R., Reichenberg, A., Patterson, T. L., Heaton, R. K., \& Harvey, P. D. (2006). Determinants of real-world functional performance in schizophrenia subjects: Correlations with cognition, functional capacity, and symptoms. American Journal of Psychiatry, 163(3), 418425. doi: 10.1176/appi.ajp.163.3.418

Bowie, C. R., Twamley, E. W., Anderson, H., Halpern, B., Patterson, T. L., \& Harvey, P. D. (2007). Self-assessment of functional status in schizophrenia. Journal of Psychiatric Research, 41(12), 1012-1018. doi: 10.1016/j.jpsychires.2006.08.003

Brandizzi, M., Valmaggia, L., Byrne, M., Jones, C., Iwegbu, N., Badger, S., ... Fusar-Poli, P. (2015). Predictors of functional outcome in individuals at high clinical risk for psychosis at six years follow-up. J Psychiatr Res, 65, 115-123. doi: 10.1016/j.jpsychires.2015.03.005

Brekke, J., Kay, D. D., Lee, K. S., \& Green, M. F. (2005). Biosocial pathways to functional outcome in schizophrenia. Schizophrenia Research, 80(2-3), 213-225. doi: 10.1016/j.schres.2005.07.008

Broome, M. E. (2000). Integrative literature reviews for the development of concepts. Em B. L. Rodgers \& K. A. Knafl (Eds.), Concept development in nursing: foundations, techniques and applications (pp. 231-50). Philadelphia (USA): W.B Saunders Company.

Burns, T., Catty, J., White, S., Becker, T., Koletsi, M., Fioritti, A., ... Group, E. (2009). The impact of supported employment and working on clinical and social functioning: Results of an international study of individual placement and support. Schizophr Bull, 35(5), 949958. doi: 10.1093/schbul/sbn024

Campellone, T. R., Sanchez, A. H., \& Kring, A. M. (2016). Defeatist performance beliefs, negative symptoms, and functional outcome in schizophrenia: A meta-analytic review. Schizophrenia Bulletin, 42(6), 1343-1352. doi: 10.1093/schbul/sbw026

Cardenas, V., Abel, S., Bowie, C. R., Tiznado, D., Depp, C. A., Patterson, T. L., ... Mausbach, B. T. (2013). When Functional Capacity and Real-World Functioning Converge: The Role of Self-Efficacy. Schizophrenia Bulletin, 39(4), 908-916. doi: 10.1093/schbul/sbs004

Cardoso, C. S., Bandeira, M., Caiaffa, W. T., \& Fonseca, J. O. P. (2002). Escala de qualidade de vida para pacientes com esquizofrenia (QLSBR): adaptação transcultural para o Brasil. Jornal Brasileiro de Psiquiatria, 51(1), 31-38.

Cassidy, C. M., Norman, R., Manchanda, R., Schmitz, N., \& Malla, A. (2010). Testing definitions of symptom remission in first-episode psychosis for prediction of functional outcome at 2 years. Schizophr Bull, 36(5), 1001-1008. doi: 10.1093/schbul/sbp007

Chan, H. M., Stolwyk, R., Neath, J., Kelso, W., Walterfang, M., Mocellin, R., ... Velakoulis, D. (2015). Neurocognitive similarities between severe chronic schizophrenia and behavioural variant frontotemporal dementia. Psychiatry Res, 225(3), 658-666. doi: 10.1016/j. psychres.2014.11.029

Ciudad, A., Olivares, J. M., Bousono, M., Gomez, J. C., \& Alvarez, E. (2006). Improvement in social functioning in outpatients with schizophrenia with prominent negative symptoms treated with olanzapine or risperidone in a 1 year randomized, open-label trial. Prog Neuropsychopharmacol Biol Psychiatry, 30(8), 1515-1522. doi: 10.1016/j.pnpbp.2006.05.010

Cornblatt, B. A., Auther, A. M., Niendam, T., Smith, C. W., Zinberg, J., Bearden, C. E., \& Cannon, T. D. (2007). Preliminary findings for two new measures of social and role functioning in the prodromal phase of schizophrenia. Schizophr Bull, 33(3), 688-702. doi: 10.1093/schbul/sbm029

Cornblatt, B. A., Carrion, R. E., Addington, J., Seidman, L., Walker, E. F., Cannon, T. D., ... Lencz, T. (2012). Risk factors for psychosis: impaired social and role functioning. Schizophr Bull, 38(6), 1247-1257. doi: 10.1093/schbul/sbr136

Cruz, B. F., Resende, C. B. de, Carvalhaes, C. F., Cardoso, C. S., Teixeira, A. L., Keefe, R. S., ... Salgado, J. V. (2016). Interview-based assessment of cognition is a strong predictor of quality of life in patients with schizophrenia and severe negative symptoms. Revista Brasileira de Psiquiatria, 38(3), 216-221. doi: 10.1590/1516-4446-2015-1776

Dickinson, D., Bellack, A. S., \& Gold, J. M. (2007). Social/communication skills, cognition, and vocational functioning in schizophrenia. Schizophr Bull, 33(5), 1213-1220. doi: 10.1093/schbul/sbl067

Dragovic, M., Fajgelj, S., \& Panickacheril John, A. (2016). Do the generalised cognitive deficits observed in schizophrenia indicate a rapidly-ageing brain? The European Journal of Psychiatry, 30(2), 141-148. Recuperado de http://scielo.isciii.es/scielo.php?script=sci arttext\&pid $=$ S0213-61632016000200005\&lng $=$ en\&nrm $=$ iso\&tlng $=$ en

Eack, S. M., Bahorik, A. L., McKnight, S. A., Hogarty, S. S., Greenwald, D. P., Newhill, C. E., ... Minshew, N. J. (2013). Commonalities in social and non-social cognitive impairments in adults with autism spectrum disorder and schizophrenia. Schizophr Res, 148(1-3), 24-28. doi: 10.1016/j.schres.2013.05.013

Eack, S. M., Pogue-Geile, M. F., Greenwald, D. P., Hogarty, S. S., \& Keshavan, M. S. (2011). Mechanisms of functional improvement in a 2-year trial of cognitive enhancement therapy for early schizophrenia. Psychol Med, 41(6), 1253-1261. doi: 10.1017/S0033291710001765

Fervaha, G., Foussias, G., Agid, O., \& Remington, G. (2015). Motivational deficits in early schizophrenia: prevalent, persistent, and key determinants of functional outcome. Schizophr Res, 166(1-3), 9-16. doi: 10.1016/j.schres.2015.04.040

Fleischhacker, W. W., Arango, C., Arteel, P., Barnes, T. R., Carpenter, W., Duckworth, K., ... Woodruff, P. (2014). Schizophrenia--time to commit to policy change. Schizophr Bull, 40 Suppl 3, S165-94. doi: 10.1093/schbul/sbu006

Frese, F. J., Knight, E. L., \& Saks, E. (2009). Recovery from schizophrenia: With views of psychiatrists, psychologists, and others diagnosed with this disorder. Schizophrenia Bulletin, 35(2), 370-380. https://doi.org/10.1093/schbul/sbn175 
Gladsjo, J. A., McAdams, L. A., Palmer, B. W., Moore, D. J., Jeste, D. V, \& Heaton, R. K. (2004). A six-factor model of cognition in schizophrenia and related psychotic disorders: relationships with clinical symptoms and functional capacity. Schizophr Bull, 30(4), 739754. Recuperado de http://www.ncbi.nlm.nih.gov/pubmed/15957196

Gorwood, P., Burns, T., Juckel, G., Rossi, A., San, L., Hargarter, L., ... Africa functioning group. (2013). Psychiatrists' perceptions of the clinical importance, assessment and management of patient functioning in schizophrenia in Europe, the Middle East and Africa. Ann Gen Psychiatry, 12(1), 8. doi: 10.1186/1744-859X-12-8

Gould, F., Sabbag, S., Durand, D., Patterson, T. L., \& Harvey, P. D. (2013). Self-assessment of functional ability in schizophrenia: Milestone achievement and its relationship to accuracy of self-evaluation. Psychiatry Research, 207(1-2), 19-24. doi: 10.1016/j.psychres.2013.02.035

Granholm, E., Ben-Zeev, D., Fulford, D., \& Swendsen, J. (2013). Ecological Momentary Assessment of social functioning in schizophrenia: impact of performance appraisals and affect on social interactions. Schizophr Res, 145(1-3), 120-124. Recuperado de http://www.ncbi. nlm.nih.gov/pubmed/23402693

Granholm, E., Ben-Zeev, D., \& Link, P. C. (2009). Social disinterest attitudes and group cognitive-behavioral social skills training for functional disability in schizophrenia. Schizophr Bull, 35(5), 874-883. doi: 10.1093/schbul/sbp072

Granholm, E., McQuaid, J. R., McClure, F. S., Auslander, L. A., Perivoliotis, D., Pedrelli, P., .. Jeste, D. V. (2005). A randomized, controlled trial of cognitive behavioral social skills training for middle-aged and older outpatients with chronic schizophrenia. Am J Psychiatry, 162(3), 520-529. doi: 10.1176/appi.ajp.162.3.520

Grant, P. M., Huh, G. A., Perivoliotis, D., Stolar, N. M., \& Beck, A. T. (2012). Randomized trial to evaluate the efficacy of cognitive therapy for low-functioning patients with schizophrenia. Arch Gen Psychiatry, 69(2), 121-127. doi: 10.1001/archgenpsychiatry.2011.129

Green, M. F., Kern, R. S., Braff, D. L., \& Mintz, J. (2000). Neurocognitive deficits and functional outcome in schizophrenia: are we measuring the "right stuff"? Schizophrenia Bulletin, 26(1), 119-136. doi: 10.1093/oxfordjournals.schbul.a033430

Green, M. F., Llerena, K., \& Kern, R. S. (2015). The "right Stuff" Revisited: What Have We Learned about the Determinants of Daily Functioning in Schizophrenia? Schizophrenia Bulletin, 41(4), 781-785. doi: 10.1093/schbul/sbv018

Harvey, P. D. (2014). Disability in schizophrenia: Contributing factors and validated assessments. Journal of Clinical Psychiatry, 75(suppl. 1), 15-20. doi: 10.4088/JCP.13049su1c.03

Harvey, P. D., \& Bellack, A. S. (2009). Toward a terminology for functional recovery in schizophrenia: Is functional remission a viable concept? Schizophrenia Bulletin, 35(2), 300-306. doi: 10.1093/schbul/sbn171

Heinrichs, D. W., Ammari, N., Miles, A. A., \& McDermid Vaz, S. (2010). Cognitive performance and functional competence as predictors of community independence in schizophrenia. Schizophr Bull, 36(2), 381-387. doi: 10.1093/schbul/sbn095

Heinrichs, D. W., Hanlon, T. E., \& Carpenter Jr., W. T. (1984). The Quality of Life Scale: an instrument for rating the schizophrenic deficit syndrome. Schizophr Bull, 10(3), 388-398. Recuperado de http://www.ncbi.nlm.nih.gov/pubmed/6474101

Horan, W. P., Green, M. F., DeGroot, M., Fiske, A., Hellemann, G., Kee, K., ... Nuechterlein, K. H. (2012). Social Cognition in Schizophrenia, Part 2: 12-Month Stability and Prediction of Functional Outcome in First-Episode Patients. Schizophrenia Bulletin, 38(4), 865-872. doi: 10.1093/schbul/sbr001

Karilampi, U., Helldin, L., \& Archer, T. (2011). Cognition and global assessment of functioning in male and female outpatients with schizophrenia spectrum disorders. J Nerv Ment Dis, 199(7), 445-448. doi: 10.1097/NMD.0b013e318221413e

Keefe, R. S. E., Poe, M., Walker, T. M., \& Harvey, P. D. (2006). The Relationship of the Brief Assessment of Cognition in Schizophrenia (BACS) to Functional Capacity and Real-world Functional Outcome. Journal of Clinical and Experimental Neuropsychology, 28(2), 260269. doi: 10.1080/13803390500360539

Keefe, R. S. E., Poe, M., Walker, T. M., Kang, J. W., \& Harvey, P. D. (2006). The schizophrenia cognition rating scale: An interview-based assessment and its relationship to cognition, real-world functioning, and functional capacity. American Journal of Psychiatry, 163(3), 426-432. doi: 10.1176/appi.ajp.163.3.426

Köhler, O., Horsdal, H. T., Baandrup, L., Mors, O., \& Gasse, C. (2016). Association between global assessment of functioning scores and indicators of functioning, severity, and prognosis in first-time schizophrenia. Clinical Epidemiology, 8, 323-332. doi: 10.2147/CLEP.S109036

Li, N., Ying, C., \& Deng, H. (2012). Cross-sectional assessment of the factors associated with occupational functioning in patients with schizophrenia. Shanghai Arch Psychiatry, 24(4), 222-230. doi: 10.3969/j.issn.1002-08329.2012.04.003

Lugnegard, T., Unenge Hallerback, M., Hjarthag, F., \& Gillberg, C. (2013). Social cognition impairments in Asperger syndrome and schizophrenia. Schizophr Res., 143(2-3), 277-284. doi: 10.1016/j.schres.2012.12.001

Lysaker, P. H., Roe, D., \& Yanos, P. T. (2006). Toward understanding the insight paradox: Internalized stigma moderates the association between insight and social functioning, hope, and self-esteem among people with schizophrenia spectrum disorders. Schizophrenia Bulletin, 33(1), 192-199. doi: 10.1093/schbul/sbl016

MacDonald, A. W., Goghari, V. M., Hicks, B. M., Flory, J. D., Carter, C. S., \& Manuck, S. B. (2005). A convergent-divergent approach to context processing, general intellectual functioning, and the genetic liability to schizophrenia. Neuropsychology, 19(6), 814-821. doi: 10.1037/0894-4105.19.6.814

Magliano, L., Fiorillo, A., Malangone, C., De Rosa, C., \& Maj, M. (2006). Patient functioning and family burden in a controlled, real-world trial of family psychoeducation for schizophrenia. Psychiatr Serv, 57(12), 1784-1791. doi: 10.1176/appi.ps.57.12.1784

Mathews, J. R., \& Barch, D. M. (2010). Emotion responsivity, social cognition, and functional outcome in schizophrenia.J Abnorm Psychol, 119(1), 50-59. doi: 10.1037/a0017861

Mausbach, B. T., Bowie, C. R., Harvey, P. D., Twamley, E. W., Goldman, S. R., Jeste, D. V., \& Patterson, T. L. (2008). Usefulness of the UCSD performance-based skills assessment (UPSA) for predicting residential independence in patients with chronic schizophrenia. Journal of Psychiatric Research, 42(4), 320-327. doi: 10.1016/j.jpsychires.2006.12.008

Mausbach, B. T., Depp, C. A., Bowie, C. R., Harvey, P. D., McGrath, J. A., Thronquist, M. H., ... Patterson, T. L. (2011). Sensitivity and specificity of the UCSD Performance-based Skills Assessment (UPSA-B) for identifying functional milestones in schizophrenia. Schizophrenia Research, 132(2-3), 165-170. doi: 10.1016/j.schres.2011.07.022

Mausbach, B. T., Harvey, P. D., Goldman, S. R., Jeste, D. V., \& Patterson, T. L. (2006). Development of a brief scale of everyday functioning in persons with serious mental illness. Schizophrenia Bulletin, 33(6), 1364-1372. doi: 10.1093/schbul/sbm014

Mausbach, B. T., Harvey, P. D., Pulver, A. E., Depp, C. A., Wolyniec, P. S., Thornquist, M. H., ... Patterson, T. L. (2010). Relationship of the Brief UCSD Performance-based Skills Assessment (UPSA-B) to multiple indicators of functioning in people with schizophrenia and bipolar disorder. Bipolar Disorders, 12(1), 45-55. doi: 10.1111/j.1399-5618.2009.00787.x

Mausbach, B. T., Moore, R., Bowie, C., Cardenas, V., \& Patterson, T. L. (2009). A Review of instruments for measuring functional recovery in those diagnosed with psychosis. Schizophrenia Bulletin, 35(2), 307-318. doi: 10.1093/schbul/sbn152 
McFarlane, W. R., Levin, B., Travis, L., Lucas, F. L., Lynch, S., Verdi, M., ... Spring, E. (2015). Clinical and functional outcomes after 2 years in the early detection and intervention for the prevention of psychosis multisite effectiveness trial. Schizophr Bull, 41(1), 30-43. doi: $10.1093 / \mathrm{schbul} / \mathrm{sbu} 108$

Moore, R. C., Harmell, A. L., Ho, J., Patterson, T. L., Eyler, L. T., Jeste, D. V, \& Mausbach, B. T. (2013). Initial validation of a computerized version of the UCSD Performance-Based Skills Assessment (C-UPSA) for assessing functioning in schizophrenia. Schizophrenia Research, 144(1-3), 87-92. doi: 10.1016/j.schres.2012.12.028

Moore, R. C., Viglione, D. J., Rosenfarb, I. S., Patterson, T. L., \& Mausbach, B. T. (2013). Rorschach measures of cognition relate to everyday and social functioning in schizophrenia. Psychological Assessment, 25(1), 253-263. doi: 10.1037/a0030546

Niendam, T. A., Bearden, C. E., Zinberg, J., Johnson, J. K., O’Brien, M., \& Cannon, T. D. (2007). The course of neurocognition and social functioning in individuals at ultra-high risk for psychosis. Schizophr Bull, 33(3), 772-781. doi: 10.1093/schbul/sbm020

Ochoa, S., Salvador-Carulla, L., Villalta-Gil, V., Gilbert, K., \& Haro, J. M. (2012). Use of functioning-disability and dependency for casemix and subtyping of schizophrenia. European Journal of Psychiatry, 26(1), 5-20. doi: 0.4321/S0213-61632012000100001

Pasquali, L. (2010). Instrumentação Psicológica: fundamentos e práticas. Porto Alegre: Artmed.

Pinkham, A. E., Penn, D. L., Green, M. F., \& Harvey, P. D. (2015). social cognition psychometric evaluation: Results of the initial psychometric study. Schizophrenia Bulletin, Advanced A, 1-11. doi: 10.1093/schbul/sbv056

Pitkanen, A., Valimaki, M., Kuosmanen, L., Katajisto, J., Koivunen, M., Hatonen, H., ... Knapp, M. (2012). Patient education methods to support quality of life and functional ability among patients with schizophrenia: a randomised clinical trial. Qual Life Res, 21(2), 247256. doi: 10.1007/s11136-011-9944-1

Pontes, L. M. M., \& Elkis, H. (2013). Treinamento de atenção e memória na esquizofrenia. Um Manual Prático. Porto Alegre: Artmed.

Rademeyer, M., \& Joubert, P. (2016). A comparison between the mini-mental state examination and the montreal cognitive assessment test in schizophrenia. South African Journal of Psychiatry, 22(1), 5. doi: 10.4102/sajpsychiatry.v22i1.890

Reeder, C., Smedley, N., Butt, K., Bogner, D., \& Wykes, T. (2006). Cognitive predictors of social functioning improvements following cognitive remediation for schizophrenia. Schizophr Bull, 32 Suppl. 1, S123-31. doi: 10.1093/schbul/sbl019

Rocha C., N. Q., Aguiar, S., \& Marques, A. (2008). Implicações da neurocognição e da auto-eficácia na predição do funcionamento psicossocial de pessoas com esquizofrenia. Análise Psicológica, 4(XXVI), 587-600.

Sabbag, S., Twamley, E. W., Vella, L., Heaton, R. K., Patterson, T. L., \& Harvey, P. D. (2012). Predictors of the accuracy of self-assessment of everyday functioning in people with schizophrenia. Schizophrenia Research, 137(1-3), 190-195. doi: 10.1016/j.schres.2012.02.002

Shi, C., He, Y., Cheung, E. F., Yu, X., \& Chan, R. C. (2013). An ecologically valid performance-based social functioning assessment battery for schizophrenia. Psychiatry Res., 210(3), 787-793. doi: 10.1016/j.psychres.2013.09.023

Silveira, C., Parpinelli, M. A., Pacagnella, R. C., Camargo, R. S., Costa, M. L., Zanardi, D. M., ... Andreucci, C. B. (2013). Adaptação transcultural da escala de avaliação de incapacidades da organizaçãomundial de saúde (WHODAS 2.0) para o Português. Revista da Associação Médica Brasileira, 59(3), 234-240. doi: 0.1016/j.ramb.2012.11.005

Thornicroft, G., \& Tansella, M. (2010). Mental health outcome measures (3rd ed.). London: RCPsych Publications.

Ustun, B., \& Kennedy, C. (2009). What is "functional impairment"? Disentangling disability from clinical significance. World Psychiatry, 8(2), 82-85. Recuperado de http://www.ncbi.nlm.nih.gov/pubmed/19516924

Vaskinn, A., Sundet, K., Simonsen, C., Hellvin, T., Melle, I., \& Andreassen, O. A. (2011). Sex differences in neuropsychological performance and social functioning in schizophrenia and bipolar disorder. Neuropsychology, 25(4), 499-510. doi: 10.1037/a0022677

Velligan, D. I., Alphs, L., Lancaster, S., Morlock, R., \& Mintz, J. (2009). Association between changes on the Negative Symptom Assessment scale (NSA-16) and measures of functional outcome in schizophrenia. Psychiatry Res., 169(2), 97-100. Doi: 10.1016/j.psychres.2008.10.009

Voruganti, L. P., Awad, A. G., Parker, G., Forrest, C., Usmani, Y., Fernando, M. L., \& Senthilal, S. (2007). Cognition, functioning and quality of life in schizophrenia treatment: results of a one-year randomized controlled trial of olanzapine and quetiapine. Schizophr Res., 96(1-3), 146-155. doi: 10.1016/j.schres.2007.08.002

Walther, S., Eisenhardt, S., Bohlhalter, S., Vanbellingen, T., M??ri, R., Strik, W., \& Stegmayer, K. (2016). Gesture Performance in Schizophrenia Predicts Functional Outcome after 6 Months. Schizophrenia Bulletin, 42(6), 1326-1333. doi: 10.1093/schbul/sbw124

World Health Organization. (2010a). International Statistical Classification of Diseases and Related Health Problems 10th Revision (ICD-10). Recuperado de http://www.who.int/classifications/icd/ICD10Volume2_en_2010.pdf?ua=1

World Health Organization. (2010b). Measuring health and disability: Manual for WHO disability assessment schedule (WHODAS 2.0). Recuperado de http://apps.who.int/iris/bitstream/10665/43974/1/9789241547598_eng.pdf?ua=1

World Health Organization, \& Cássia Maria Buchalla (Eds.). (2003). İEF: International Classification of Functioning, Disability and Health. Recuperado de http://apps.who.int/iris/bitstream/10665/42407/111/9788531407840_por.pdf?ua=1

Wykes, T., \& Reeder, C. (2005). Cognitive remediation therapy for schizophrenia: Theory and practice. London; New York: Routledge.

Zawadzki, J. A., Girard, T. A., Foussias, G., Rodrigues, A., Siddiqui, I., Lerch, J. P., ... Wong, A. H. (2013). Simulating real world functioning in schizophrenia using a naturalistic city environment and single-trial, goal-directed navigation. Front Behav Neurosci, 7, 180. doi: 10.3389/fnbeh.2013.00180

Zimmer, M., Duncan, A. V, Laitano, D., Ferreira, E. E., \& Belmonte-de-Abreu, P. (2007). A twelve-week randomized controlled study of the cognitive-behavioral Integrated Psychological Therapy program: Positive effect on the social functioning of schizophrenic patients. Revista Brasileira de Psiquiatria, 29(2), 140-147. doi: 0.1590/S1516-44462006005000030

\section{Sobre os autores}

Luciana Amorim é Mestre e Doutoranda em avaliação psicológica, Universidade Federal de Santa Catarina.

Luciano Franzin Neto é Doutorando em avaliação psicológica, Universidade Federal de Santa Catarina.

Aline Battisti Archer é Mestre e Doutoranda em Psicologia Organizacional e do Trabalho, Universidade Federal de Santa Catarina. Bolsista CAPES.

Juliana Frainer é Mestre em avaliação psicológica, Universidade Federal de Santa Catarina.

Roberto Moraes Cruz. PhD é Docente do programa de pós graduação em Psicologia da UFSC. 